\title{
Significant association between CYP3A5 polymorphism and blood concentration of tacrolimus in patients with connective tissue diseases
}

\author{
Kosuke Tanaka ${ }^{1}$, Chikashi Terao ${ }^{1}$, Koichiro Ohmura ${ }^{2}$, Meiko Takahashi ${ }^{1}$, Ran Nakashima ${ }^{2}$, Yoshitaka Imura ${ }^{2}$, \\ Hajime Yoshifuji ${ }^{2}$, Naoichiro Yukawa ${ }^{2}$, Takashi Usui ${ }^{2}$, Takao Fujii ${ }^{2}$, Tsuneyo Mimori ${ }^{2}$ and Fumihiko Matsuda ${ }^{1}$ \\ Although the association between CYP3A5 polymorphism and blood concentration of tacrolimus (TAC) in patients with solid \\ organ transplantation was established, whether the association is also true in patients with connective tissue disease (CTD) who \\ usually receive small amount of TAC is uncertain. Here, we performed a quantitative linear regression analysis to address the \\ association between CYP3A5 and blood TAC concentration in patients with CTD. A total of 72 patients with CTD were recruited \\ in the current study and genotyped for rs776746 in CYP3A5, which showed strong association with TAC concentration in \\ patients with solid organ transplantation. The blood trough concentration of TAC after taking $3 \mathrm{mg}$ per day was retrospectively \\ obtained for each patient. As a result, allele A of rs776746 showed a significant association with a decreasing blood \\ concentration of TAC $(P=0.0038)$. Those who are carrying at least one copy of the A allele displayed decreased mean \\ concentration of TAC by $31.0 \%$ compared with subjects with GG genotype. Rs 776746 is associated with concentrations of \\ TAC in patients with CTD.
}

Journal of Human Genetics (2014) 59, 107-109; doi:10.1038/jhg.2013.129; published online 19 December 2013

Keywords: connective tissue disease; pharmacogenetics; tacrolimus

Tacrolimus (FK506, TAC) is a calcineurin inhibitor isolated from Streptomyces tsukubaensis ${ }^{1}$ and one of the many types of powerful immunosuppressants that are frequently used for solid organ transplantation to prevent organ rejection. ${ }^{2}$ TAC is also used for patients with connective tissue disease (CTD) including rheumatoid arthritis (RA), systemic lupus erythematosus, polymyositis and dermatomyositis to control disease activity. ${ }^{3,4}$ TAC is metabolized mainly by cytochrome P450 (CYP) $3 \mathrm{~A}$ in the liver and intestine. ${ }^{5}$ Because TAC concentration is highly variable among patients, to predict TAC concentration to achieve therapeutic effect is a big challenge. Previous studies revealed that the variation of TAC concentration is largely attributable to different expressions of CYP3A in patients of organ transplantation. Patients carrying $C Y P 3 A 5^{\star} 3$ determined by the $\mathrm{G}$ allele of rs776746 were shown to have high TAC concentration than patients with the A allele. ${ }^{5,6}$ Although TAC is a substrate for P-glycoprotein encoded by the $A B C B 1$ gene, effects of polymorphisms in $A B C B 1$ on TAC concentration are inconclusive. ${ }^{6-8}$ Genetic studies have been performed mainly recruiting patients with organ transplantation to date. The number of previous studies focusing on TAC concentration in non-organ transplantation subjects is limited..$^{9,10}$
When TAC is given to patients with CTD, the dosage is around $3 \mathrm{mg}$ per day, ${ }^{3,4}$ which is much lower than that given to patients of organ transplantation. For example, patients with renal transplantation receive $0.3 \mathrm{mg} \mathrm{kg}^{-1}$ per day at the transplantation and $0.12 \mathrm{mg} \mathrm{kg}^{-1}$ per day as maintainance. ${ }^{11}$ In addition, although recipients of renal transplantation take TAC twice daily, ${ }^{11}$ patients with only CTD take a single dose of TAC per day. The effect of CYP3A5 on TAC concentrations with low TAC exposure in patients with CTD has not been studied so far. Furthermore, chronic, systemic and autoimmune inflammatory process in CTD may influence the metabolism and concentration of TAC. Thus, whether the association between polymorphisms of CYP3A5 and TAC concentrations can be observed in patients with CTD remained unclear. Here, we performed an association study to address this point.

This study was designed in accordance with the Helsinki Declaration and approved by the ethics committee of Kyoto University Graduate School and Faculty of Medicine. A total of 72 subjects with CTD who were prescribed to take a single dose of $3 \mathrm{mg}$ of TAC every day in the evening at the Department of Rheumatology and Clinical Immunology, Kyoto University Graduate School of Medicine from December 2005 to December 2012 were enrolled in this study. Written informed consent was obtained from all the participants. Patients

${ }^{1}$ Center for Genomic Medicine, Kyoto University Graduate School of Medicine, Kyoto, Japan and ${ }^{2}$ Department of Rheumatology and Clinical Immunology, Kyoto University Graduate School of Medicine, Kyoto, Japan

Correspondence: Dr C Terao, Center for Genomic Medicine, Kyoto University Graduate School of Medicine, Kyoto 606-8507, Japan. E-mail: a0001101@kuhp.kyoto-u.ac.jp

Received 26 September 2013; revised 25 November 2013; accepted 25 November 2013; published online 19 December 2013 
took PROGRAF (Astellas Pharma Inc., Tokyo, Japan) capsules containing 1 or $0.5 \mathrm{mg}$ TAC. CTD patients fulfilled criteria for each disease, namely, American College of Rheumatology (ACR) criteria for RA in $1987^{12}$ or ACR/EULAR criteria in $2010,{ }^{13}$ for systemic lupus erythematosus, ${ }^{14}$ polymyositis and dermatomyositis ${ }^{15}$ and for polyarteritis nodosa and microscopic polyangiitis. ${ }^{16}$ Information of age, sex, weight, serum creatinine and date of prescription, dosage, and blood concentration of TAC were obtained from clinical charts retrospectively by the system previously described. ${ }^{17}$ Information of prescription and dosage of corticosteroid was also obtained. TAC concentration data were obtained at least one week after prescription was initiated or changed. The data of concurrent use of cyclosporine or bosentan, contraindication due to interaction with TAC, was excluded. Estimated glomelular filtration ratio (eGFR) was inferred by serum creatinine, age and sex. The blood trough TAC concentration with $3 \mathrm{mg}$ TAC (around $12 \mathrm{~h}$ after taking TAC) was used. TAC concentrations were quantified by two measurements according to measuring time; namely, microparticle enzyme immunoassay (MEIA, IMxTM-TACRO II, Abbott Laboratories, Green Oaks, IL, USA) until May 2009 and chemiluminescent immunoassay (CLIA, ARCHITECT_TACRO, Abbott Laboratories) from May 2009. These measurements can quantify even low TAC concentrations $\left(\sim 1.5\right.$ and $\sim 0.5 \mathrm{ng} \mathrm{ml}^{-1}$, respectively). When TAC concentrations were available for both measurements in a patient, data of CLIA with lower measuring limit of TAC concentrations was used. A total of 63 and 9 patients were quantified by CLIA and MEIA, respectively (hereafter termed as CLIA group and MEIA group, respectively). When multiple TAC concentrations were available, the mean of them was adopted. Calculations were performed on the basis of logarithm of TAC concentrations to obtain normal distribution and to avoid excess influence of extreme data.

The summary of the subjects in the current study is shown in Table 1. Log-transformation of TAC concentration supported applying linear regression analysis. When we analyzed correlations between TAC concentration and age, sex, weight, eGFR, dosage or usage of corticosteroid or the presence of RA or systemic lupus erythematosus by single linear regression analysis, none of them displayed overall significant associations $(P \geqslant 0.083)$. However, because presence of RA showed a suggestive association in MEIA group $(P=0.0091)$, we used presence of RA as a covariate. Rs776746, whose $G$ allele determined CYP $3 A 5^{\star} 3$, was selected on the basis of previous studies and genotyped by the Taqman assay (Applied Biosystems Inc, Foster city, CA, USA). As a result, no deviation from Hardy-Weinberg equilibrium was observed $(P=0.13)$. Although previous reports comparing different measurement methods of blood concentration of TAC showed good correlations $(r \geqslant 0.84)$ and did not detect discrepancy even in ranges of low concentrations, ${ }^{18,19}$ MEIA was suggested to underestimate TAC concentrations in low levels. ${ }^{19}$ Thus we analyzed the associations in CLIA and MEIA groups separately, and the overall

Table 1 Summary of subjects in the current study

\begin{tabular}{|c|c|}
\hline & Study subjects \\
\hline Age $^{a}$ & $48.94 \pm 17.24$ \\
\hline Sex & Male 13, female 59 \\
\hline Disease & $\begin{array}{l}\text { RA: } 22, \text { SLE: } 43, \mathrm{DM}: 3 \text {, } \\
\text { PM: 2, PAN: } 1, \mathrm{mPA}: 1\end{array}$ \\
\hline
\end{tabular}

associations were estimated by meta-analysis using inverse-variance method. We found a significant decreasing effect of the A allele of rs776746 on TAC concentration ( $P=0.0038$, Figure 1$)$. Both MEIA and CLIA groups showed the comparable effect sizes, supporting the accuracy of the result (Table 2). Patients who carried A allele had $31.0 \%$ lower mean concentration than those who were homozygote for $\mathrm{G}$ allele. Although the current and the previous studies ${ }^{11}$ showed a good fit of the dose-dependent model of rs776746, there are also conflicting reports. ${ }^{6,20}$ Considering the limited number of subjects with AA genotype, the dose-dependent effect of rs776746 should be regarded as inconclusive. Meta-analysis of the recessive model resulted in a comparable result $(P=0.0035, \mathrm{AA}+\mathrm{GA}$ vs $\mathrm{GG})$.

The current study provided evidence that TAC concentration was strongly influenced by CYP3A5 in patients with CTD even taking a small amount of TAC. Our results showed the same direction of A allele of rs776746 and comparable effect sizes in the previous studies using patients of solid organ transplantation., ${ }^{5,6}$ Disease-specific influence on TAC concentrations was not clear. As this study contained relatively small number of subjects and low TAC concentrations around the measurement limits might be associated with diminished accuracy, these results should be replicated by a larger number of patients with CTD, also including other populations. Because the predictive model of TAC concentration is proposed in

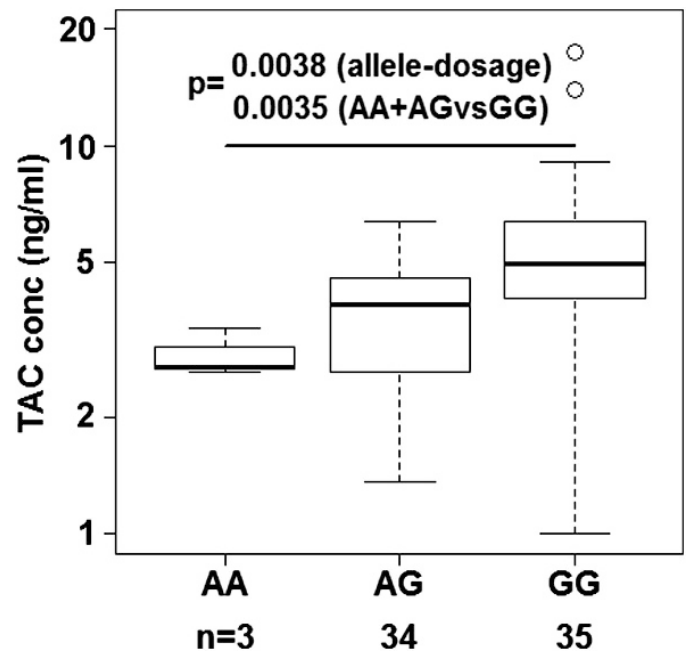

Figure 1 Association between TAC concentration and the polymorphism in CYP3A5 in patients with CTD. The obtained or inferred TAC concentrations adjusted for $3 \mathrm{mg}$ TAC are shown according to rs776746 genotypes. $Y$ axis is shown in log scale. The mean concentrations are $2.88,3.57$ and 5.10 ng $\mathrm{ml}^{-1}$ for $A A, A G$ and $G G$ genotypes, respectively. TAC concentrations were adjusted for MEIA group.

Table 2 Association between rs776746 and TAC concentration in multiple regression analysis

\begin{tabular}{lclll}
\hline & Number & Beta & s.e. & P-value \\
\hline CLIA & 63 & 0.106 & 0.041 & 0.012 \\
MEIA & 9 & 0.161 & 0.12 & 0.22 \\
Overall & 72 & 0.112 & 0.039 & 0.0038
\end{tabular}

Abbreviations: CLIA, chemiluminescent immunoassay; MEIA, microparticle enzyme immunoassay; TAC, tacrolimus.

Statistics adjusted by rheumatoid arthritis presence. 
patients of organ transplantation, it will be interesting to construct a predictive model of TAC concentration in patients with CTD.

\section{CONFLICT OF INTEREST}

The authors declare no conflict of interest.

\section{ACKNOWLEDGEMENTS}

We would like to thank all the patients registered in this study. This study was supported by Kyoto University Step-up grant.

1 Kino, T., Hatanaka, H., Miyata, S., Inamura, N., Nishiyama, M., Yajima, T. et al. FK-506, a novel immunosuppressant isolated from a Streptomyces. II. Immunosuppressive effect of FK-506 in vitro. J. Antibiot. (Tokyo) 40, 1256-1265 (1987).

2 Scott, L. J., McKeage, K., Keam, S. J. \& Plosker, G. L. Tacrolimus: a further update of its use in the management of organ transplantation. Drugs 63, 1247-1297 (2003).

3 Takeuchi, T., Kawai, S., Yamamoto, K., Harigai, M., Ishida, K. \& Miyasaka, N. Post-marketing surveillance of the safety and effectiveness of tacrolimus in 3,267 Japanese patients with rheumatoid arthritis. Mod. Rheumatol. 24, 8-16 (2014).

4 Takahashi, S., Hiromura, K., Sakurai, N., Matsumoto, T., Ikeuchi, H., Maeshima, A. et al. Efficacy and safety of tacrolimus for induction therapy in patients with active lupus nephritis. Mod. Rheumatol. 21, 282-289 (2011).

5 Hesselink, D. A., van Gelder, T. \& van Schaik, R. H. The pharmacogenetics of calcineurin inhibitors: one step closer toward individualized immunosuppression? Pharmacogenomics 6, 323-337 (2005).

6 Tsuchiya, N., Satoh, S., Tada, H., Li, Z., Ohyama, C., Sato, K. et al. Influence of CYP3A5 and MDR1 (ABCB1) polymorphisms on the pharmacokinetics of tacrolimus in renal transplant recipients. Transplantation 78, 1182-1187 (2004).

7 Tada, H., Tsuchiya, N., Satoh, S., Kagaya, H., Li, Z., Sato, K. et al. Impact of CYP3A5 and MDR1(ABCB1) C3435T polymorphisms on the pharmacokinetics of tacrolimus in renal transplant recipients. Transplant. Proc. 37, 1730-1732 (2005).

8 Vafadari, R., Bouamar, R., Hesselink, D. A., Kraaijeveld, R., van Schaik, R. H., Weimar, W. et al. Genetic polymorphisms in ABCB1 influence the pharmacodynamics of tacrolimus. Ther. Drug. Monit. 35, 459-465 (2013).

9 Hirai, F., Takatsu, N., Yano, Y., Satou, Y., Takahashi, H., Ishikawa, S. et al. CYP3A5 genetic polymorphisms affect the pharmacokinetics and short-term remission in patients with ulcerative colitis treated with tacrolimus. J. Gastroenterol. Hepatol. (in press).

10 Shi, X. J., Geng, F., Jiao, Z., Cui, X. Y., Qiu, X. Y. \& Zhong, M. K. Association of ABCB1, CYP3A $4 * 18 B$ and CYP $3 A 5 * 3$ genotypes with the pharmacokinetics of tacrolimus in healthy Chinese subjects: a population pharmacokinetic analysis. J. Clin. Pharm. Ther. 36, 614-624 (2011).

11 Niioka, T., Kagaya, H., Miura, M., Numakura, K., Saito, M., Inoue, T. et al. Pharmaceutical and genetic determinants for interindividual differences of tacrolimus bioavailability in renal transplant recipients. Eur. J. Clin. Pharmacol. 69, 1659-1665 (2013).

12 Arnett, F. C., Edworthy, S. M., Bloch, D. A., McShane, D. J., Fries, J. F., Cooper, N. S. et al. The American Rheumatism Association 1987 revised criteria for the classification of rheumatoid arthritis. Arthritis. Rheum. 31, 315-324 (1988).

13 Aletaha, D., Neogi, T., Silman, A. J., Funovits, J., Felson, D. T., Bingham, C. O. 3rd et al. Rheumatoid arthritis classification criteria: an American College of Rheumatology/European League Against Rheumatism collaborative initiative. Arthritis. Rheum. 62, 2569-2581 (2010).

14 Hochberg, M. C. Updating the American College of Rheumatology revised criteria for the classification of systemic lupus erythematosus. Arthritis. Rheum. 40, 1725 (1997).

15 Bohan, A. \& Peter, J. B. Polymyositis and dermatomyositis (first of two parts). N. Engl. J. Med. 292, 344-347 (1975).

16 Bloch, D. A., Michel, B. A., Hunder, G. G., McShane, D. J., Arend, W. P., Calabrese, L. H. et al. The American College of Rheumatology 1990 criteria for the classification of vasculitis. Patients and methods. Arthritis. Rheum. 33, 1068-1073 (1990).

17 Terao, C., Hashimoto, M., Yamamoto, K., Murakami, K., Ohmura, K., Nakashima, R. et al. Three groups in the 28 joints for rheumatoid arthritis synovitis-Analysis using more than 17,000 assessments in the KURAMA database. PLoS One 8, e59341 (2013).

18 Ju, M. K., Chang, H. K., Kim, H. J., Huh, K. H., Ahn, H. J., Kim, M. S. et al. Is the affinity column-mediated immunoassay method suitable as an alternative to the microparticle enzyme immunoassay method as a blood tacrolimus assay? Transplant. Proc. 40, 3673-3678 (2008).

19 Marubashi, S., Nagano, H., Kobayashi, S., Eguchi, H., Takeda, Y., Tanemura, M. et al. Evaluation of a new immunoassay for therapeutic drug monitoring of tacrolimus in adult liver transplant recipients. J. Clin. Pharmacol. 50, 705-709 (2010).

20 Hesselink, D. A van Schaik, R. H van der Heiden, I. P van der Werf, M. Gregoor, P. J., Lindemans, J. et al. Genetic polymorphisms of the CYP3A4, CYP3A5, and MDR- 1 genes and pharmacokinetics of the calcineurin inhibitors cyclosporine and tacrolimus. Clin. Pharmacol. Ther. 74, 245-254 (2003). 